Network Working Group

J. Rosenberg

Request for Comments: 5079

$\mathrm{Cisco}$

Category: Standards Track

December 2007

Rejecting Anonymous Requests in the Session Initiation Protocol (SIP)

Status of This Memo

This document specifies an Internet standards track protocol for the Internet community, and requests discussion and suggestions for improvements. Please refer to the current edition of the "Internet Official Protocol Standards" (STD 1) for the standardization state and status of this protocol. Distribution of this memo is unlimited.

Abstract

The Session Initiation Protocol (SIP) allows for users to make anonymous calls. However, users receiving such calls have the right to reject them because they are anonymous. SIP has no way to indicate to the caller that the reason for call rejection was that the call was anonymous. Such an indication is useful to allow the call to be retried without anonymity. This specification defines a new SIP response code for this purpose.

Table of Contents

1. Introduction . . . . . . . . . . . . . . . . . . . . . . . . 2

2. Terminology . . . . . . . . . . . . . . . . . . . . . . 2

3. Server Behavior . . . . . . . . . . . . . . . . . . . 3

4. UAC Behavior . . . . . . . . • . . . . . . . . . . . . . . . . 4

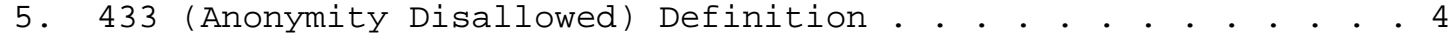

6. IANA Considerations . . . . . . . . . . . . . . . . . . . . 4 4

7. Security Considerations . . . . . . . . . . . . . . . . 5

8. Acknowledgements . . . . . . . . . . . . . . . . . . 5

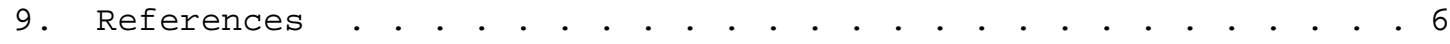
9.1. Normative References . . . . . . . . . . . . . . 6 9.2. Informative References . . . . . . . . . . . . . . . 6 


\section{Introduction}

The Session Initiation Protocol (SIP) [RFC3261] allows for users to make anonymous calls. In RFC 3261, this is done by including a From header field whose display name has the value of "Anonymous". Greater levels of anonymity were subsequently defined in [RFC3323], which introduces the Privacy header field. The Privacy header field allows a requesting User Agent (UA) to ask for various levels of anonymity, including user level anonymity, header level anonymity, and session level anonymity. [RFC3325] additionally defined the P-Asserted-Identity header field, used to contain an asserted identity. RFC 3325 also defined the 'id' value for the Privacy header field, which is used to request the network to remove the P-Asserted-Identity header field.

Though users need to be able to make anonymous calls, users that receive such calls retain the right to reject the call because it is anonymous. SIP does not provide a response code that allows the User Agent Server (UAS), or a proxy acting on its behalf, to explicitly indicate that the request was rejected because it was anonymous. The closest response code is 403 (Forbidden), which doesn't convey a specific reason. While it is possible to include a reason phrase in a 403 response that indicates to the human user that the call was rejected because it was anonymous, that reason phrase is not useful for automata and cannot be interpreted by callers that speak a different language. An indication that can be understood by an automaton would allow for programmatic handling, including user interface prompts, or conversion to equivalent error codes in the Public Switched Telephone Network (PSTN) when the client is a gateway .

To remedy this, this specification defines the 433 (Anonymity Disallowed) response code.

2. Terminology

The key words "MUST", "MUST NOT", "REQUIRED", "SHALL", "SHALL NOT", "SHOULD", "SHOULD NOT", "RECOMMENDED", "MAY", and "OPTIONAL" in this document are to be interpreted as described in [RFC2119]. 


\section{Server Behavior}

A server (generally acting on behalf of the called party, though this need not be the case) MAY generate a 433 (Anonymity Disallowed) response when it receives an anonymous request, and the server refuses to fulfill the request because the requestor is anonymous. A request SHOULD be considered anonymous when the identity of the originator of the request has been explicitly withheld by the originator. This occurs in any one of the following cases:

- The From header field contains a URI within the anonymous.invalid domain.

- The From header field contains a display name whose value is either 'Anonymous' or 'anonymous'. Note that display names make a poor choice for indicating anonymity, since they are meant to be consumed by humans, not automata. Thus, language variations and even misspelling can cause an automaton to miss a hint in the display name. Despite these problems, a check on the display name is included here because RFC 3261 explicitly calls out the usage of the display name as a way to declare anonymity.

- The request contained a Privacy header field whose value indicates that the user wishes its identity withheld. Values meeting this criteria are 'id' [RFC3325] or 'user'.

- The From header field contains a URI that has an explicit indication that it is anonymous. One such example of a mechanism that would meet this criteria is [coexistence]. This criteria is true even if the request has a validated Identity header field [RFC4474], which can be used in concert with anonymized From header fields.

Lack of a network-asserted identity (such as the P-Asserted-Identity header field), in and of itself, SHOULD NOT be considered an indication of anonymity. Even though a Privacy header field value of 'id' will cause the removal of a network-asserted identity, there is no way to differentiate this case from one in which a networkasserted identity was not supported by the originating domain. As a consequence, a request without a network-asserted identity is considered anonymous only when there is some other indication of this, such as a From header field with a display name of 'Anonymous'.

In addition, requests where the identity of the requestor cannot be determined or validated, but it is not a consequence of an explicit action on the part of the requestor, are not considered anonymous. For example, if a request contains a non-anonymous From header field, along with the Identity and Identity-Info header fields [RFC4474], 
but the certificate could not be obtained from the reference in the Identity-Info header field, it is not considered an anonymous request, and the 433 response code SHOULD NOT be used.

4. UAC Behavior

A User Agent Client (UAC) receiving a 433 (Anonymity Disallowed) MUST NOT retry the request without anonymity unless it obtains confirmation from the user that this is desirable. Such confirmation could be obtained through the user interface, or by accessing userdefined policy. If the user has indicated that this is desirable, the UAC MAY retry the request without requesting anonymity. Note that if the UAC were to automatically retry the request without anonymity in the absence of an indication from the user that this treatment is desirable, then the user's expectations would not be met. Consequently, a user might think it had completed a call anonymously when it is not actually anonymous.

Receipt of a 433 response to a mid-dialog request SHOULD NOT cause the dialog to terminate, and SHOULD NOT cause the specific usage of that dialog to terminate [RFC5057].

A UAC that does not understand or care about the specific semantics of the 433 response will treat it as a 400 response.

5. 433 (Anonymity Disallowed) Definition

This response indicates that the server refused to fulfill the request because the requestor was anonymous. Its default reason phrase is "Anonymity Disallowed".

6. IANA Considerations

This section registers a new SIP response code according to the procedures of RFC 3261 .

RFC Number: RFC 5079

Response Code Number: 433

Default Reason Phrase: Anonymity Disallowed 


\section{Security Considerations}

The fact that a request was rejected because it was anonymous does reveal information about the called party -- that the called party does not accept anonymous calls. This information may or may not be sensitive. If it is, a UAS SHOULD reject the request with a 403 instead.

In the Public Switched Telephone Network (PSTN), the Anonymous Call Rejection (ACR) feature is commonly used to prevent unwanted calls from telemarketers (also known as spammers). Since telemarketers frequently withhold their identity, anonymous call rejection has the desired effect in many (but not all) cases. It is important to note that the response code described here is likely to be ineffective in blocking SIP-based spam. The reason is that a malicious caller can include a From header field and display name that is not anonymous, but is meaningless and invalid. Without a Privacy header field, such a request will not appear anonymous and thus not be blocked by an anonymity screening service. Dealing with sIP-based spam is not a simple problem. The reader is referred to [sipping-spam] for a discussion of the problem.

When anonymity services are being provided as a consequence of an anonymizer function acting as a back-to-back user agent (B2BUA) [RFC3323], and the anonymizer receives a 433 response, the anonymizer MUST NOT retry the request without anonymization unless it has been explicitly configured by the user to do so. In essence, the same rules that apply to a UA in processing of a 433 response apply to a network-based anonymization function, and for the same reasons.

8. Acknowledgements

This document was motivated based on the requirements in [tispan-req], and has benefited from the concepts in [hautakorpi]. Thanks to Keith Drage, Paul Kyzivat, and John Elwell for their reviews of this document. 


\title{
9. References
}

\subsection{Normative References}

[RFC3261] Rosenberg, J., Schulzrinne, H., Camarillo, G., Johnston, A., Peterson, J., Sparks, R., Handley, M., and E. Schooler, "SIP: Session Initiation Protocol", RFC 3261, June 2002 .

[RFC3323]

Peterson, J., "A Privacy Mechanism for the Session Initiation Protocol (SIP)", RFC 3323, November 2002.

[RFC2119] Bradner, S., "Key words for use in RFCs to Indicate Requirement Levels", BCP 14, RFC 2119, March 1997.

[RFC4474] Peterson, J. and C. Jennings, "Enhancements for Authenticated Identity Management in the Session Initiation Protocol (SIP)", RFC 4474, August 2006.

\subsection{Informative References}

\author{
$[\mathrm{RFC} 3325]$ \\ Jennings, C., Peterson, J., and M. Watson, "Private \\ Extensions to the Session Initiation Protocol (SIP) \\ for Asserted Identity within Trusted Networks", \\ RFC 3325, November 2002 . \\ [coexistence] Rosenberg, J., "Coexistence of P-Asserted-ID and SIP \\ Identity", Work in Progress, June 2006. \\ [tispan-req] Jesske, R., "Input Requirements for the Session \\ Initiation Protocol (SIP) in support for the \\ European Telecommunications Standards Institute", \\ Work in Progress, July 2007. \\ [hautakorpi] Hautakorpi, J. and G. Camarillo, "Extending the \\ Session Initiation Protocol Reason Header with \\ Warning Codes", Work in Progress, October 2005. \\ [RFC5057] Sparks, R., "Multiple Dialog Usages in the Session \\ Initiation Protocol", RFC in 5057, November 2007. \\ [sipping-spam] Jennings, C. and J. Rosenberg, "The Session \\ Initiation Protocol (SIP) and Spam", Work \\ in Progress, August 2007.
}


Author's Address

Jonathan Rosenberg

Cisco

Edison, NJ

US

EMail: jdrosendcisco.com

URI : http://www.jdrosen. net 
Full Copyright statement

Copyright (C) The IETF Trust (2007).

This document is subject to the rights, licenses and restrictions contained in BCP 78, and except as set forth therein, the authors retain all their rights.

This document and the information contained herein are provided on an "AS IS" basis and THE CONTRIBUTOR, THE ORGANIZATION HE/SHE REPRESENTS OR IS SPONSORED BY (IF ANY), THE INTERNET SOCIETY, THE IETF TRUST AND THE INTERNET ENGINEERING TASK FORCE DISCLAIM ALL WARRANTIES, EXPRESS OR IMPLIED, INCLUDING BUT NOT LIMITED TO ANY WARRANTY THAT THE USE OF THE INFORMATION HEREIN WILL NOT INFRINGE ANY RIGHTS OR ANY IMPLIED WARRANTIES OF MERCHANTABILITY OR FITNESS FOR A PARTICULAR PURPOSE.

Intellectual Property

The IETF takes no position regarding the validity or scope of any Intellectual Property Rights or other rights that might be claimed to pertain to the implementation or use of the technology described in this document or the extent to which any license under such rights might or might not be available; nor does it represent that it has made any independent effort to identify any such rights. Information on the procedures with respect to rights in RFC documents can be found in BCP 78 and BCP 79 .

Copies of IPR disclosures made to the IETF Secretariat and any assurances of licenses to be made available, or the result of an attempt made to obtain a general license or permission for the use of such proprietary rights by implementers or users of this specification can be obtained from the IETF on-line IPR repository at http://www.ietf.org/ipr.

The IETF invites any interested party to bring to its attention any copyrights, patents or patent applications, or other proprietary rights that may cover technology that may be required to implement this standard. Please address the information to the IETF at ietf-ipreietf.org. 